\title{
Misexpression of AtTX12 encoding a Toll/interleukin-1 receptor domain induces growth defects and expression of defense-related genes partially independently of EDS1 in Arabidopsis
}

Sang-Kee Song*

Department of Biology, Chosun University, Gwangju 61452, Korea
In this study, a tissue-specific GAL4/UAS activation tagging system was used for the characterization of genes which could induce lethality when ubiquitously expressed. A dominant mutant exhibiting stunted growth was isolated and named defective root development 1-D (drd1-D). The T-DNA tag was located within the promoter region of AtTX12, which is predicted to encode a truncated nucleotide-binding leucinerich repeat (NLR) protein, containing a Toll/interleukin-1 receptor (TIR) domain. The transcript levels of AtTX12 and defense-related genes were elevated in $\operatorname{drd1}-D$, and the misexpression of AtTX12 recapitulated the drd1-D phenotypes. In the presence of ENHANCED DISEASE SUSCEPTIBILITY 1 (EDS1), a key transducer of signals triggered by TIR-type NLRs, a low-level of AtTX12 misexpression induced strong defective phenotypes including seedling lethality whereas, in the absence of EDS1, a high-level of AtTX12 misexpression induced weak growth defects like dwarfism, suggesting that AtTX12 might function mainly in an EDS1-dependent and partially in an EDS1-independent manner. [BMB Reports 2016; 49(12): 693-698]

\section{INTRODUCTION}

To survive as sessile organisms, plants exhibit enormous plasticity in growth and development in response to various external cues, including biotic and abiotic stresses. Biotic stresses mainly originate from plant pathogens and herbivores,

${ }^{*}$ Corresponding author. Tel: +82-62-230-6655; Fax: +82-62-2306650; E-mail: sangkeesong@chosun.ac.kr

https://doi.org/10.5483/BMBRep.2016.49.12.180

Received 24 October 2016, Revised 28 October 2016, Accepted 2 November 2016

Keywords: Arabidopsis thaliana, EDS1-dependent defense-related gene expression, EDS1-independent defense-related gene expression, Stunted growth, Toll/Interleuin-1 receptor domain protein against which plants have evolved a wide range of defense mechanisms. Plants protect themselves from invasion by biotrophic or hemibiotrophic pathogens through innate immune responses. Resistance against non-specific pathogens is mediated by pattern recognition receptors which can detect conserved molecules such as bacterial flagellin and elongation factor Tu and lead to pathogen-associated molecular patterntriggered immunity (PTI). However, host-specific pathogens have evolved the ability to deliver effector molecules into host cells, which allow them to evade PTI. Plants have co-evolved diverse resistance $(R)$ proteins in response that sense these effectors or the host proteins that are modified by the effectors. $\mathrm{R}$ proteins induce stronger, pathogen-specific resistance responses, and are part of what is known as effector-triggered immunity (ETI), which restricts pathogen proliferation via local programmed cell death, also known as the hypersensitive response $(1,2)$.

Most $\mathrm{R}$ proteins are intracellular immune receptors with nucleotide binding (NB) and leucine-rich repeat (LRR) domains (NLR), possessing either a Toll/interleukin-1 receptor (TIR) or coiled-coil (CC) domain at the N-terminus $(3,4)$. NLRs with TIR domains (TNLs) require ENHANCED DISEASE SUSCEPTIBILITY 1 (EDS1), a eukaryotic lipase-like protein, and PHYTOALEXIN DEFICIENT 4 (PAD4), an EDS1-interacting partner, for downstream immune responses, including salicylic acid (SA)-mediated signaling, whereas most NLRs with a coiled-coil (CC) domain (CNLs) require NON-RACE-SPECIFIC DISEASE RESISTANCE 1 (NDR1) for immune signaling (5-8). TIR domains are reported to serve as platforms for homo- or hetero-dimerization to induce cell death signaling or effector perception (9-11). The NB domains of NLRs function as internal switches for conformational changes, depending on the status of the bound nucleotide. In the activated state, TIR/CC domains can be exposed to trigger defense signals. Overexpression of TIR regions of TNLs lacking NB domains induced constitutively active immune responses in the absence of effectors (11-14).

In Arabidopsis Col-0, there are 92 TNLs, 23 truncated TIR-NBs (TNs) lacking LRR domains, and 30 TIR-unknown domain (TX) proteins lacking both NB and LRR domains (15). 
The functions of a few TX genes have been characterized by stable/transient overexpression $(16,17)$. It is an intriguing question to answer whether or not the uncharacterized TXs possess activity for defense-related gene expression and follow typical TNL-mediated signaling to broaden our understanding of plant defense-related systems.

Activation tagging screening is an efficient way to characterize the unknown functions of redundant genes. Conventional activation tagging systems use viral enhancers to strongly and ubiquitously induce the expression of tagged genes (18), whereas the GAL4/UAS system (19) enhances flanking gene expression in a tissue-specific manner, which is advantageous for screening genes that induce lethality when ubiquitously expressed.

In this study, a UAS-tagged defective root development 1-D (drd1-D) mutant displaying stunted growth was isolated, and the tagged gene was identified as AtTX12, which encodes a truncated TNL lacking both the NB and LRR domains. Genetic analyses have shown that AtTX12-mediated growth defects and defense-related gene expression are independent of PAD4/NDR1 and partially independent of EDS1.

\section{RESULTS}

Isolation of the drd1-D and localization of the tagged gene A dominant mutant exhibiting arrested growth named defective root development 1-D (drd1-D) was isolated by introducing UAS enhancers into the Q2610 enhancer trap line. The putative homozygous drd1-D was seedling lethal, displaying little post-embryonic development (Fig. 1A, C) compared to wild type (WT) (Fig. 1B, D) as the Q2610 is highly active in post-embryonic roots (19).

Thermal asymmetric interlaced (TAIL)-polymerase chain reaction (PCR) analysis revealed the DNA sequences between the T-DNA and genomic DNA, which are shown in Fig. 1E. The T-DNA, which contains UAS elements, was located 215 bp upstream of the putative start codon of At2g03300, previously designated as AtTX12, encoding a 203-amino acid (aa) truncated TNL containing a TIR domain and a 59-aa extension (17). The genome of the Col-0 contains AtTX11 (At2g03030), which is the closest homologue of AtTX12 probably originated from local gene duplication (15). The TIR domain of AtTX12 shares 34-54\% aa-sequence identity with those of TNLs such as RPS4, RPP1, N, and L10, and contains 4 conserved TIR motifs (Supplementary Fig. 1) (15). AtTX12-like proteins are conserved among species of the Brassicaceae family, with $66-94 \%$ identity over the entire sequence (Supplementary Fig. 2).

Reverse transcription (RT)-PCR analysis showed the increased expression of AtTX12 in drd1-D compared to that in WT (Fig. 1F). It was also examined whether the transcript levels of defense-related genes increased in $\operatorname{drd} 1-D$ as $T X$-overexpressing plants are known to exhibit elevated defense-related responses $(16,17)$. The transcripts of defense- related genes such as PATHOGENESIS-RELATED 1 (PR1), $\beta$-1,3-GLUCANASE 2 (BGL2) (20), EDS1, and PAD4 were highly accumulated in $d r d-1 D$ compared to those in WT (Fig. $1 \mathrm{~F})$, suggesting that AtTX12 may induce the expression of downstream defense-related genes.

As shown in Fig. 1G, endogenous AtTX12 was expressed at a very low level in the roots and leaves, and tended to increase as the plants matured. To examine the subcellular localization of AtTX12, transgenic plants expressing AtTX12pro:AtTX12-GFP were screened based on the semi-

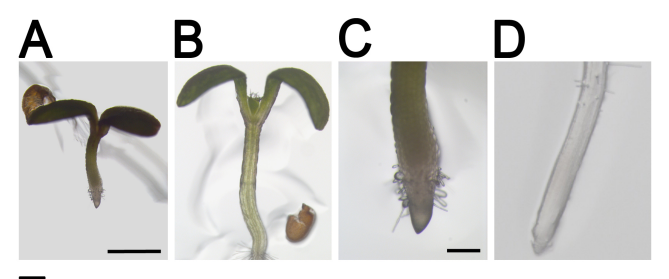

E

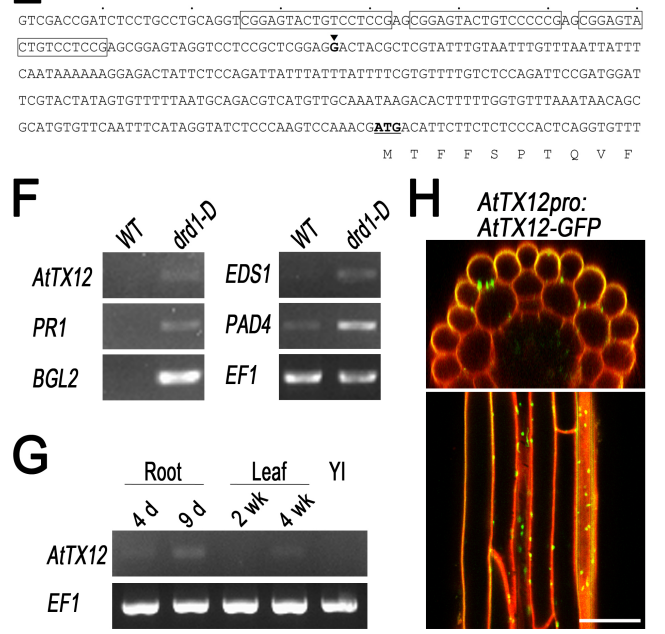

Fig. 1. The drd1-D exhibits stunted growth, which is induced by the misexpression of AtTX12. (A, B) The above-ground phenotypes of $\operatorname{drd} 1-D(\mathrm{~A})$ and WT (B). Scale bar $=1 \mathrm{~mm}$. (C, D) The root tip phenotypes of $\operatorname{drd1-D}(\mathrm{C})$ and $\mathrm{WT}(\mathrm{D})$. Scale bar $=200 \mu \mathrm{m}$. (E) The T-DNA insertion site in $d r d 1-D$ is located within the AtTX12 promoter. The 3 UAS elements in the T-DNA insert and the 5' start point of the AtTX12 promoter are shown in boxes and with an arrowhead, respectively. The putative start codon of AtTX12 is shown in boldface and underlined. (F) RT-PCR analysis of defense-related gene expression in 4-d-old drd1-D seedlings. EF1 was used as a control for the 25-cycle PCR. (G) RT-PCR analysis of organ-specific AtTX12 expression in roots of 4 and 9-d-old seedlings grown in $0.5 \times \mathrm{MS}$ media, 2- and 4-wk-old leaves, and young inflorescence (YI). EF1 was used as a control for the 35-cycle PCR. (H) Subcellular localization of an AtTX12GFP translational fusion protein expressed by a 1.8-kb AtTX12 promoter. GFP signals were mainly detected in the plasma membrane and plasmodesmata connecting neighboring cells in the root as shown in the composite cross section (upper panel) and transverse section (lower panel) images. Scale bar $=40 \mu \mathrm{m}$. 
dwarf to dwarf phenotypes. RT-PCR analysis showed that the expression of AtTX12 was well-correlated with the phenotypic strength (Supplementary Fig. 3A, 3B). AtTX12-GFP signal was found in either the plasma membrane or plasmodesmata connecting neighboring cells in the root $($ Fig. $1 \mathrm{H})$, suggesting that the AtTX12 might function in such subcellular regions.

\section{Misexpression of $A t T X 12$ recapitulates the $d r d-1 D$ phenotype} To further confirm that the drd1-D phenotype is induced by misexpression of AtTX12, transgenic plants harboring AtTX12 under the regulation of the $5 \times$ UAS promoter (UASpro:AtTX12) were screened and crossed to Q2610 to generate a double hemizygous plant for Q2610 and UASpro:AtTX12 (Q2610/+>> AtTX12/+). Q2610/+>>AtTX12/+ displayed seedling lethality (Fig. 2A), phenocopying the homozygous $d r d 1-D$, indicating that the $d r d 1-D$ phenotype originated from the misexpression of AtTX12. Two representative transgenic lines exhibited almost the same phenotypes displaying local cell death and pigment accumulation around the root tips (Fig. 2B, C), although Q2610/+ > >AtTX12/+ line \#2 accumulated a little more AtTX12 transcripts than line \#1 (Fig. 2F). RT-PCR analysis showed increased expression of defense-related genes, such as PR1, EDS1, and PAD4 in the Q2610/+>>AtTX12/+ seedlings (Fig. 2F). These results suggest that the transcript level of AtTX12 in Q2610/+> > AtTX12/+ line \#1 might be enough to induce seedling lethality.

To test whether the AtTX12 misexpression phenotype is temperature-sensitive or not, Q2610/+>>AtTX12/+ line \#2 seedlings were grown at $28^{\circ} \mathrm{C}$ as TNL-mediated growth defects are often suppressed at a higher temperature (21). As shown in Fig. 2D, no phenotypic change of seedlings harboring transgenes was observed at a high-temperature condition.

To determine whether AtTX11 induces growth defects like AtTX12, transgenic UASpro:AtTX11 lines were prepared and crossed to Q2610. The Q2610/+>>AtTX11/+ seedlings also exhibited seedling lethality (Fig. 2E).

To test whether tissue or organ-specific growth inhibition is induced by AtTX12, AtTX12 was expressed by various enhancers. $\mathrm{J1721/+}>>$ AtTX12/+ line \#2 displayed root defects lacking a root tip as $/ 1721$ is highly expressed in the columella root cap and protoxylem during embryogenesis and the phenotype was not recovered at $28^{\circ} \mathrm{C}$ (Fig. 2G, H). Q0990/+ > > AtTX12/+ line \#2 exhibited early termination of primary root growth as Q0990 is expressed in the stele initials at a low level, but even this relatively weak phenotype was not rescued at $28^{\circ} \mathrm{C}$ (Fig. 2l, J). Q0990/+> > AtTX12/+ line \#1 survived and produced double homozygous Q0990>>AtTX12 line \#1 (Fig. 2K). When AtTX12 was expressed under the regulation of the PISTILLATA (PI) promoter, which is reported to be almost petal-specifically active (22), $P I /+>>$ AtTX12/+ line \#1 displayed suppressed petal and stamen development, mimicking the pi phenotype, whereas the development of neighboring floral organs was almost unaffected (Fig. 2L, 2M) suggesting that AtTX12 expression could induce tissue-specific local cell death.

\section{Defense-related gene expression induced by AtTX12 misexpression is mainly dependent on EDS1 and partially independent of EDS1}

EDS1/PAD4 and NDR1 are generally required for TNL- and CNL-mediated defense-related responses, respectively. It was tested whether the AtTX12 activity is dependent on EDS1,
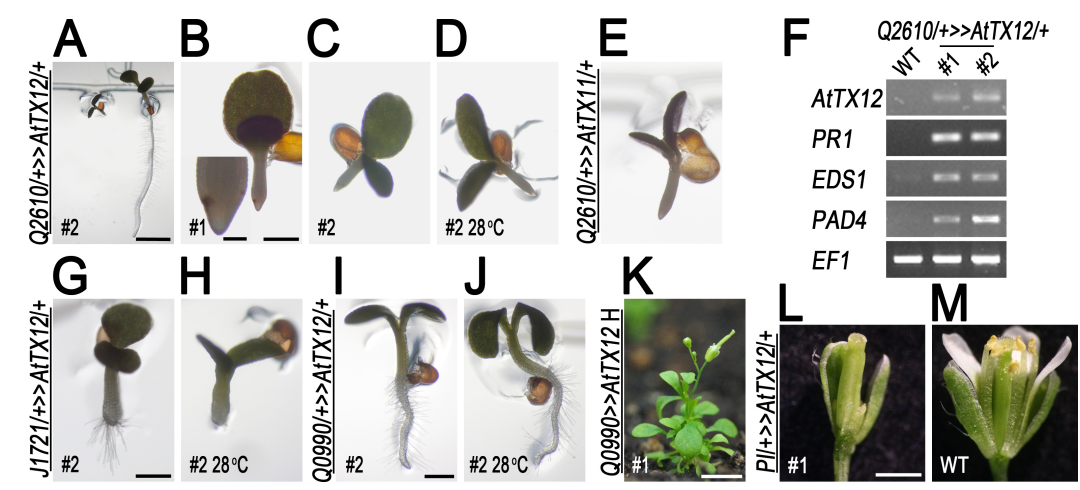

Fig. 2. Ectopic expression of $A t T X 12 / 11$ recapitulates the drd1-D phenotype by inducing defense-related gene expression. (A-D) Stunted growth phenotypes of double hemizygous Q2610/+> >AtTX12/+ seedlings $4 \mathrm{~d}$ after germination. (A) A Q2610/+>>AtTX12/+ (left) and WT-like sibling (right) of the $F_{1}$ progenies of UASpro:AtTX12 line \#2 crossed to Q2610. (B) A Q2610/+>>AtTX12/+ line \#1 seedling exhibiting local cell death and pigment accumulation. The inset shows an enlarged root tip. (C, D) Q2610/+ > >AtTX12/+ line \#2 seedlings grown at $22^{\circ} \mathrm{C}$ (C) and at $28^{\circ} \mathrm{C}$ (D). (E) A 4-d-old Q2610/+>>AtTX11/+ seedling. (F) RT-PCR analysis of AtTX12, PR1, EDS1, PAD4, and EF1 expression in 4-d-old $Q 2610 /+>>A t T X 12 /+$ line \#1 and \#2 seedlings in 25-cycle reactions. EF1 was used as a control. $(\mathrm{G}, \mathrm{H}) / 1721 /+>>$ AtTX12/+ line \#2 seedlings grown at $22^{\circ} \mathrm{C}(\mathrm{G})$ and $28^{\circ} \mathrm{C}(\mathrm{H}) .(\mathrm{I}, \mathrm{J}) \mathrm{Q} 0990 /+>>$ AtTX12/+ line \#2 seedlings grown at $22^{\circ} \mathrm{C}$ (I) and $28^{\circ} \mathrm{C}(\mathrm{J})$. (K) The dwarf phenotype of a 5-wk-old double homozygous Q0990>>AtTX12 line \#1. (L, M) Defective petal and stamens of PI/ $+>>$ AtTX12/+ line \#1 (L) and a WT-like flower (M). A sepal was removed to expose the inner whorls. Scale bars $=5 \mathrm{~mm}(\mathrm{~A}), 0.5 \mathrm{~mm}(\mathrm{~B}-\mathrm{E}), 0.1 \mathrm{~mm}$ (inset of panel $\mathrm{B}), 0.5 \mathrm{~mm}(\mathrm{G}, \mathrm{H}), 0.5 \mathrm{~mm}(\mathrm{I}, \mathrm{J}), 5 \mathrm{~mm}(\mathrm{~K})$, and $1 \mathrm{~mm}(\mathrm{~L}, \mathrm{M})$. 
PAD4, or NDR1. Q2610/+>>AtTX12/+ line \#2 pad4-1 exhibited a seedling-lethal phenotype (Fig. 3A), suggesting that PAD4 may not be essential for AtTX12-triggered growth defects (Fig 3A). RT-PCR analysis showed that AtTX12 misexpression led to increased transcript accumulation of defense-related genes in the pad4-1 background (Fig. 3B). The seedling-lethal phenotype was consistently observed when Q2610/+ > > AtTX12/+ line \#2 was introduced into 2 different putative pad4 mutants, SALK 206216 and SALK 206548 (data not shown). Q2610/+>>AtTX12/+ line \#2 ndr1-1 also exhibited a seedling-lethal phenotype. These results suggest that AtTX12 might function PAD4- and NDR1-independently (Fig. 3A).

As there is an additional isoform (At3g48080) of EDS1 (At3g48090) in the genome of the Col-0, the T-DNA insertion mutants eds1-22 (SALK_071051) and eds1-23 (SALK_057149), which originated from $\mathrm{Col}-0$, still possess partial EDS1 activity, whereas eds 1-2, which originated from the Ler possessing a single copy of EDS1, could be regarded as a null mutant. In this study, eds 1-2 mutant introgressed into Col-0 (23) was used for the genetic analyses. Seedlings of Q2610/+ > > AtTX12/+ line \#2 in the eds1-22, eds1-23, and eds1-2 backgrounds all displayed partially rescued, moderately short (approximately $65-70 \%$ of WT), weakly hairless, and twisted root phenotypes (Fig. 3A). However, at later developmental stages, Q2610/+>> AtTX12/ + line \#2 eds1-22 and eds1-23 exhibited dwarf, semisterile, and leaf chlorosis phenotypes, whereas Q2610/+ > >
AtTX12/+ line \#2 eds1-2 did not exhibit any apparent growth defective phenotype (Fig. 3C). These results suggest that the EDS1 isoform, At3g48080 may be more active or abundant in above-ground organs than in the roots and better transduce signals triggered by AtTX12 in these organs.

Therefore, the plant homozygous for both Q2610 and UASpro:AtTX12 line \#2 (Q2610>>AtTX12 line \#2) was obtained only in the eds1-2 mutant background at the $\mathrm{F}_{4}$ generation by examining the presence of Q2610-GFP and the UASpro:AtTX12. Q2610>>AtTX12 line \#2 eds1-2 displayed a shorter and more twisted root phenotype (Fig. 3A) compared to $\mathrm{Q} 2610 /+>>A t T X 12 /+$ line \#2 eds1-2, suggesting that AtTX12 may function in an expression level-dependent manner. This tendency was also found in line $\# 1$, as the Q2610/+ > > AtTX12/+ line \#1 eds1-2 displayed little defect (data not shown), whereas homozygous Q2610>>AtTX12 line \#1 eds1-2 displayed moderate root defects (Fig. 3A). These results suggest that when highly expressed, AtTX12 may induce growth defects even in the absence of EDS1 through a putative EDS1-independent pathway.

RT-PCR analysis of Q2610/+ > > AtTX12/+ line \#2 eds1-22 and eds 1-23 showed increased expression of defense-related genes, such as $P R 1$ and BGL2 possibly due to the presence of the EDS1 isoform transducing the defense-related signals efficiently from the relatively small amount of AtTX12 transcripts, whereas Q2610/+>>AtTX12/+ line \#2 eds1-2 showed little defense-related gene expression due to the
A
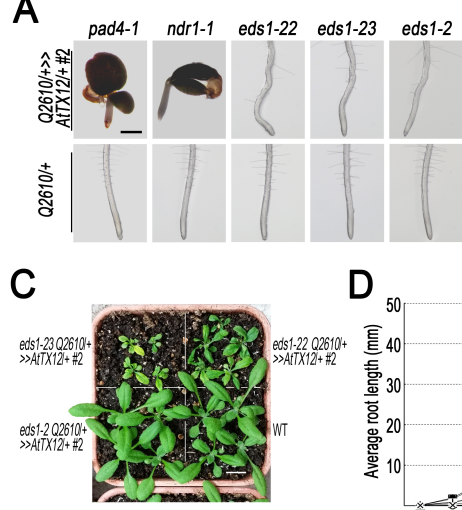

D

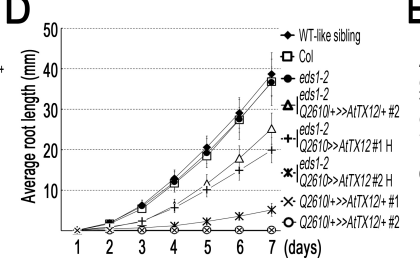

B

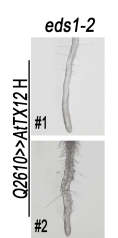

E

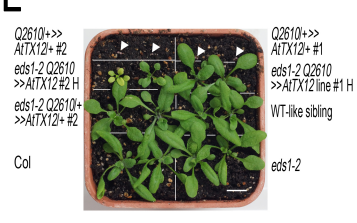

Fig. 3. The growth inhibition activity of AtTX12 is independent of PAD4/NDR1 and partially independent of EDS1. (A) Left, root tip phenotypes of 4-d-old seedlings of double hemizygous Q2610/+>>AtTX12/+ line \#2 (upper lanes) and Q2610/+ controls (lower lanes) in various plant defense-related signaling mutants, including pad4-1, ndr1-1, eds1-22, eds1-23, and eds1-2.; Right, root tips of double homozygous Q2610>>AtTX12 line \#1 and \#2 in eds1-2. Scale bar $=0.5 \mathrm{~mm}$. (B) RT-PCR analysis of AtTX12, PR1, BGL2, and EF1 expression in 4-d-old Q2610/+>>AtTX12/+ line \#2 pad4-1 seedlings (left), 2-wk-old above-ground tissues of Q2610/+ $>>$ AtTX12/+ line \#2 eds1-2, 1-22, and 1-23 with a WT control (middle) in a 25-cycle reaction, and 1-wk-old above-ground tissues of Q2610 $>>$ AtTX12 line \#1 and \#2 in eds1-2 in a 30-cycle reactions (right). (C) Above-ground phenotypes of Q2610/+ $>>$ AtTX12/+ line \#2 eds1-2, eds1-22, and eds1-23 along with WT controls at 3 wks. after germination. Scale bar = $10 \mathrm{~mm}$. (D) Average root length of eds1-2 or WT seedlings that misexpressed transgenic AtTX12 (UASpro:AtTX12 line \#1 or line \#2), hemizygously or homozygously with Q2610, together with controls of Col, eds1-2, and WT-like siblings. The root length shown is an average of 8 seedlings. Error bars indicate SD. (E) Above-ground phenotypes of 3-wk-old plants described in panel (D). Scale bar $=10 \mathrm{~mm}$. White lines were drawn to delineate plants of different genetic backgrounds $(C$ and $E)$. 
absence of any functional EDS1 thereby withstood the increased expression of AtTX12 and remained healthy (Fig. 3B, $3 C)$. The increase of the $P R 1 / B G L 2$ expression was detected in the Q2610>>AtTX12 line \#2 eds1-2 at a relatively higher number of PCR cycles (Fig. 3B), suggesting that the PR1/BGL2 expression induced by AtTX12 misexpression may be mainly dependent on EDS1 and partially independent of EDS1.

AtTX12-mediated growth inhibition in eds1-2 was wellcorrelated with the AtTX12 transcript level, as displayed in the root growth curve. Q2610>>AtTX12 line \#2 eds1-2 developed shorter roots compared with Q2610/+>>AtTX12/+ line \#2 eds1-2 and Q2610>>AtTX12 line \#1 eds1-2 (Fig. 3D, Supplementary Fig. 4). Furthermore, only the Q2610>> AtTX12 line \#2 eds1-2 developed leaf chlorosis and a dwarf phenotype in the above-ground organs (Fig. 3E) which correlate well with increased PR1/BGL2 gene expression (Fig. 3B). Together, these results further support the idea that AtTX12 can induce growth defects and defense-related gene expression partially EDS1-independently in a dose-dependent manner.

\section{DISCUSSION}

It is an intriguing question how plants defend themselves against a myriad of plant pathogens using a limited number of $R$ genes. In addition to the canonical NLRs, there exist a number of truncated NLRs, such as TNs and TXs of which the roles in defense-related gene expression have not been well characterized with a few exceptions $(16,17,24)$. In this study, it was shown that AtTX12, containing a TIR domain plus a short extension, could trigger defense-related gene expression possibly providing more diverse mechanisms for growth regulation and/or plant defense.

The AtTX12-GFP signal was mostly observed in either plasma membrane or plasmodesmata (Fig. $1 \mathrm{H}$ ) although AtTX12 is predicted to possess a putative nuclear localization signal (NLS; KKKRKDCKCELPDLKKSRTKK) at the C-terminus (aa 182-202) (25). Therefore, it is needed to be tested whether this putative NLS is required for AtTX12 activity as reported in RPS4 (26).

Endogenous levels of AtTX12 expression did not affect plant growth. However, when AtTX12 expression exceeds a threshold level, the excessive AtTX12 is likely to induce growth defects and defense-related gene expression in an EDS1-dependent or -independent manner. In the presence of functional EDS1, low levels AtTX12 misexpression can efficiently induce a defective growth phenotype together with defense-related gene expression (Fig. 2F), leading to a strong seedling-lethal phenotype (Fig. 2B). In contrast, in the absence of EDS1, only high levels of AtTX12 misexpression can induce growth defects, albeit less efficiently with weakly increased defense-related gene expression (Fig. 3B), resulting in weaker defective phenotypes, such as dwarfism and leaf chlorosis (Fig. $3 \mathrm{E})$. The growth defects induced by AtTX12 in the absence of EDS1 might be transduced through PAD4/SENESCENCE
ASSOCIATED GENE 101 (27) or, as yet unknown molecules present in the $\mathrm{C} 24$ ecotype, with lower efficiency compared to EDS1. As shown in Fig. 3A and C, misexpression of AtTX12 in the pad4-1 background induced growth defects together with defense-related gene expression, which is partially correlated with the previous observation that PAD4 is not required for the local acute response induced by TNLs but is required for basal immune responses (28).

Together, it is concluded that AtTX12 could induce growth defects and defense-related gene expression mainly in an EDS1-dependent and partially in an EDS1-independent way.

\section{MATERIALS AND METHODS}

\section{Plant materials and growth conditions}

GAL4/UAS enhancer trap lines, pad4-1, 2 pad4 T-DNA insertion mutants (SALK 206548 and SALK 206216), ndr1-1, and eds1-22 (SALK 071051) were obtained from the Arabidopsis Biological Resource Center (USA). Col eds1-2 (23) and eds1-23 (SALK 057149) were kindly provided by Tae-Houn Kim (Duksung Women's University, Korea). The Q2610 line used in this study was selected based on the non-late flowering phenotype after backcrossing to Col-0. Plants were grown as described in the supplementary materials.

\section{Mutant screening and determination of the T-DNA insertion site \\ UAS-tagged mutants from the $\mathrm{T}_{1}$ pool of Q2610 transformed with the pBIB-UAS vector kindly provided by Keiji Nakajima (NAIST, Japan) (19) were screened on $0.5 \times$ MS medium containing hygromycin $\mathrm{B}$ and then moved to normal medium for observation. TAIL-PCR was performed as described in the Supplementary Materials and Methods.}

Preparation of gene constructs and genotyping markers Gene constructs and genotyping markers were prepared as described in the Supplementary Materials and Methods.

\section{Gene expression analyses}

For the RT-PCR analysis, $1 \mu \mathrm{g}$ of total RNA extracted from the various tissues of WT, mutant, and transgenic plants at various developmental stages was used for RT as described previously (29) and PCR amplified with gene-specific primers for AtTX12 (5'-GACTAGTATGACATTCTTCTCTCCCACT-3' and 5'-TACTA GTTCACAACTTTTTGGTTCTGCTT-3') and other genes as described previously (16).

\section{Confocal microscopy}

The AtTX12-GFP signal was detected with a Zeiss LSM700 confocal microscope after seedlings were stained as described previously (29). 


\section{ACKNOWLEDGEMENTS}

This work was supported by the National Research Foundation of Korea (grant no. NRF-2015R1D1A1A01059797) and research funds from Chosun University 2014 (K206888001) and 2015 (K206888002) to S.S.

\section{REFERENCES}

1. DeYoung BJ and Innes RW (2006) Plant NBS-LRR proteins in pathogen sensing and host defense. Nat Immunol 7, 1243-1249

2. Jones JD and Dangl JL (2006) The plant immune system. Nature 444, 323-329

3. Meyers BC, Dickerman AW, Michelmore RW, Sivaramakrishnan S, Sobral BW and Young ND (1999) Plant disease resistance genes encode members of an ancient and diverse protein family within the nucleotide-binding superfamily. Plant J 20, 317-332

4. Meyers BC (2003) Genome-Wide Analysis of NBS-LRREncoding Genes in Arabidopsis. Plant Cell 15, 809-834

5. Falk A, Feys BJ, Frost LN, Jones JD, Daniels MJ and Parker JE (1999) EDS1, an essential component of $\mathrm{R}$ genemediated disease resistance in Arabidopsis has homology to eukaryotic lipases. Proc Natl Acad Sci U S A 96, 3292-3297

6. Jirage D, Tootle TL, Reuber TL et al (1999) Arabidopsis thaliana PAD4 encodes a lipase-like gene that is important for salicylic acid signaling. Proc Natl Acad Sci U S A 96, 13583-13588

7. Aarts N, Metz M, Holub E, Staskawicz BJ, Daniels MJ and Parker JE (1998) Different requirements for EDS1 and NDR1 by disease resistance genes define at least two $R$ gene-mediated signaling pathways in Arabidopsis. Proc Natl Acad Sci U S A 95, 10306-10311

8. Wiermer M, Feys BJ and Parker JE (2005) Plant immunity: the EDS1 regulatory node. Curr Opin Plant Biol 8, 383-389

9. Bernoux M, Ve T, Williams S et al (2011) Structural and functional analysis of a plant resistance protein TIR domain reveals interfaces for self-association, signaling, and autoregulation. Cell Host Microbe 9, 200-211

10. Williams SJ, Sohn KH, Wan L et al (2014) Structural basis for assembly and function of a heterodimeric plant immune receptor. Science 344, 299-303

11. Sukarta OC, Slootweg EJ and Goverse A (2016) Structureinformed insights for NLR functioning in plant immunity. Semin Cell Dev Biol 56, 134-149

12. Frost $D$, Way $H$, Howles $P$ et al (2004) Tobacco transgenic for the flax rust resistance gene $L$ expresses allele-specific activation of defense responses. Mol Plant Microbe Interact 17, 224-232

13. Swiderski MR, Birker D and Jones JD (2009) The TIR domain of TIR-NB-LRR resistance proteins is a signaling domain involved in cell death induction. Mol Plant Microbe Interact 22, 157-165

14. Michael Weaver L, Swiderski MR, Li Y and Jones JD
(2006) The Arabidopsis thaliana TIR-NB-LRR R-protein, RPP1A; protein localization and constitutive activation of defence by truncated alleles in tobacco and Arabidopsis. Plant J 47, 829-840

15. Meyers BC, Morgante M and Michelmore RW (2002) TIR-X and TIR-NBS proteins: two new families related to disease resistance TIR-NBS-LRR proteins encoded in Arabidopsis and other plant genomes. Plant J 32, 77-92

16. Kato H, Saito T, Ito H, Komeda $Y$ and Kato A (2014) Overexpression of the TIR-X gene results in a dwarf phenotype and activation of defense-related gene expression in Arabidopsis thaliana. J Plant Physiol 171, 382-388

17. Nandety RS, Caplan JL, Cavanaugh K et al (2013) The role of TIR-NBS and TIR-X proteins in plant basal defense responses. Plant Physiol 162, 1459-1472

18. Weigel D, Ahn JH, Blazquez MA et al (2000) Activation tagging in Arabidopsis. Plant Physiol 122, 1003-1013

19. Waki T, Miyashima $S$, Nakanishi M, Ikeda $Y$, Hashimoto $T$ and Nakajima K (2013) A GAL4-based targeted activation tagging system in Arabidopsis thaliana. Plant J 73, 357367

20. Uknes S, Mauch-Mani B, Moyer M et al (1992) Acquired resistance in Arabidopsis. Plant Cell 4, 645-656

21. Alcazar R and Parker JE (2011) The impact of temperature on balancing immune responsiveness and growth in Arabidopsis. Trends Plant Sci 16, 666-675

22. Burgos-Rivera B and Dawe RK (2012) An Arabidopsis tissue-specific RNAi method for studying genes essential to mitosis. PLoS One 7, e51388

23. Bartsch M, Gobbato E, Bednarek P et al (2006) Salicylic acid-independent ENHANCED DISEASE SUSCEPTIBILITY1 signaling in Arabidopsis immunity and cell death is regulated by the monooxygenase FMO1 and the Nudix hydrolase NUDT7. Plant Cell 18, 1038-1051

24. Zhao T, Rui L, Li J et al (2015) A truncated NLR protein, TIR-NBS2, is required for activated defense responses in the exo70B1 mutant. PLoS Genet 11, e1004945

25. Nguyen Ba AN, Pogoutse A, Provart N and Moses AM (2009) NLStradamus: a simple Hidden Markov Model for nuclear localization signal prediction. BMC Bioinformatics 10,202

26. Wirthmueller L, Zhang Y, Jones JD and Parker JE (2007) Nuclear accumulation of the Arabidopsis immune receptor RPS4 is necessary for triggering EDS1-dependent defense. Curr Biol 17, 2023-2029

27. Feys BJ, Wiermer M, Bhat RA et al (2005) Arabidopsis SENESCENCE-ASSOCIATED GENE101 stabilizes and signals within an ENHANCED DISEASE SUSCEPTIBILITY1 complex in plant innate immunity. Plant Cell 17, 2601-2613

28. Rietz S, Stamm A, Malonek S et al (2011) Different roles of Enhanced Disease Susceptibility1 (EDS1) bound to and dissociated from Phytoalexin Deficient4 (PAD4) in Arabidopsis immunity. New Phytol 191, 107-119

29. Kang YH, Kirik V, Hulskamp M et al (2009) The MYB23 gene provides a positive feedback loop for cell fate specification in the Arabidopsis root epidermis. Plant Cell 21, 1080-1094 